\title{
Case Study about efficient AI(Artificial Intelligence) Implementation Strategy
}

\author{
JeongBeom Kim \\ Namseoul University, Head Professor of BigData Master Degree Dept., Daehak Ro, \\ Sunghwaneup, Choongnam, Korea \\ jbkim@nsu.ac.kr
}

\begin{abstract}
The main purpose of this paper is to study about efficient AI project implementation as a case study. For the purpose of successful AI implementation, the project plan should be robust with long term view. This implementation strategy include top management decision, organization and human resource, infra structure for AI system, end user support, and company strategy. In this study, the performance index in analyzed from the view point of ROI and business priority.
\end{abstract}

Keywords: AI, Case Study, Implementation, RPA, Big Data Analysis

\section{Introduction}

Artificial Intelligence (AI) is a field of computer science that focuses on solving cognitive problems, primarily related to human intelligence, such as learning, problem solving, and pattern recognition. Artificial intelligence, commonly abbreviated to "AI", may be robotics or the future, but $\mathrm{AI}$ is becoming a reality of advanced computer engineering beyond the small robots in science fiction novels. . With the recent improvements in statistical computing efficiency, Bayesian has successfully developed several areas in the field of "machine learning". Likewise, as network computing continues to evolve, neural network study can further develop sub-disciplines under the name "deep learning." Both Machine Learning (ML) and Deep Learning (DL) are computer science fields that are derived from artificial intelligence. RPA(Robot Process Automation) is a field of process automation using AI technology which can replace human works.[1]

\section{Related Study}

Machine learning is the name that often applies to some Bayesian techniques used for pattern recognition and learning. Machine learning is a collection of algorithms that learns from and predicts based on recorded data, optimizes basic utility functions under uncertainty, extracts hidden structures from data, and categorizes data into concise descriptions. Machine learning is mainly deployed when explicit programming is too strict or not practical. Machine learning uses data to generate statistical codes (ML models), unlike general computer code, which software developers develop to produce output by program code based on a given input. Deep running is a type of neural network that is one of the machine running. Neural networks have

\footnotetext{
${ }^{1}$ Article history:

Received (April 9, 2019), Review Result (May 6, 2019), Accepted (June 2 , 2019)
} 
been studied since the 10950s, and may have been studied or used during school days. Neural networks are machine learning algorithms that model neurons in living organisms as they are called neurons. Deep Learning is based on the use of deep-running auto-encoders, which Jeffrey Hinton published in 2006, for preliminary learning of multilayer neural networks. After learning dropout and activation function, which can be called learning study, it became possible to learn realistic calculation amount of neural network with several hidden layers. Deep running is a machine learning using a multi-layer neural network, and new detours are still appearing. We look forward to seeing what neural networks are coming. Introducing feature quantities that characterize data in machine learning is a very effective means. There are various types of learning methods in machine learning.[2]

Map learning is a learning method that learns the correctness and correctness of each data when learning. The goal is to be able to output appropriate classification and numerical values in unknown data as a result of learning. Bid map learning only uses data that does not have correct answer information. You do not need the correct answer and only have the data, so you can collect the learning data easily. You can use it for exploratory work because it can be used at the unknown stage. It is difficult to equip the learning data with the correct answer. On the other hand, in non-word learning, there is no need for correct answers and it is easy to gather data, but there is little or no way to reflect the desired intent. Thus, there is also learning of a ring that progresses learning with data having a small amount of correct answer to data that does not add a large amount of correct answers. Finally, reinforcement learning is a learning process in which the system is rewarded by the environment according to the result of selecting an action, which improves the behavior and adapts to the environment through trial and error.[3]

As the 4th industrial revolution is emerging in all industry, many companies and government organizations have been implementing big data system project with high expectation. Also the technology level of AI(Artificial Intelligence) is getting improving, similar projects are being implemented. These days many companies are keenly interested in RPA (Robotic Process Automation). RBA is one of the AI implementation method which is getting popular in many successful cases. RPA, which was a solution that some vendors have been emphasizing, Beyond the digital innovation organization, senior executives, and even the working staff often referred to as the 'generic' technology. From finance and manufacturing to distribution, to food, to public, it is a popular phenomenon. [4] 


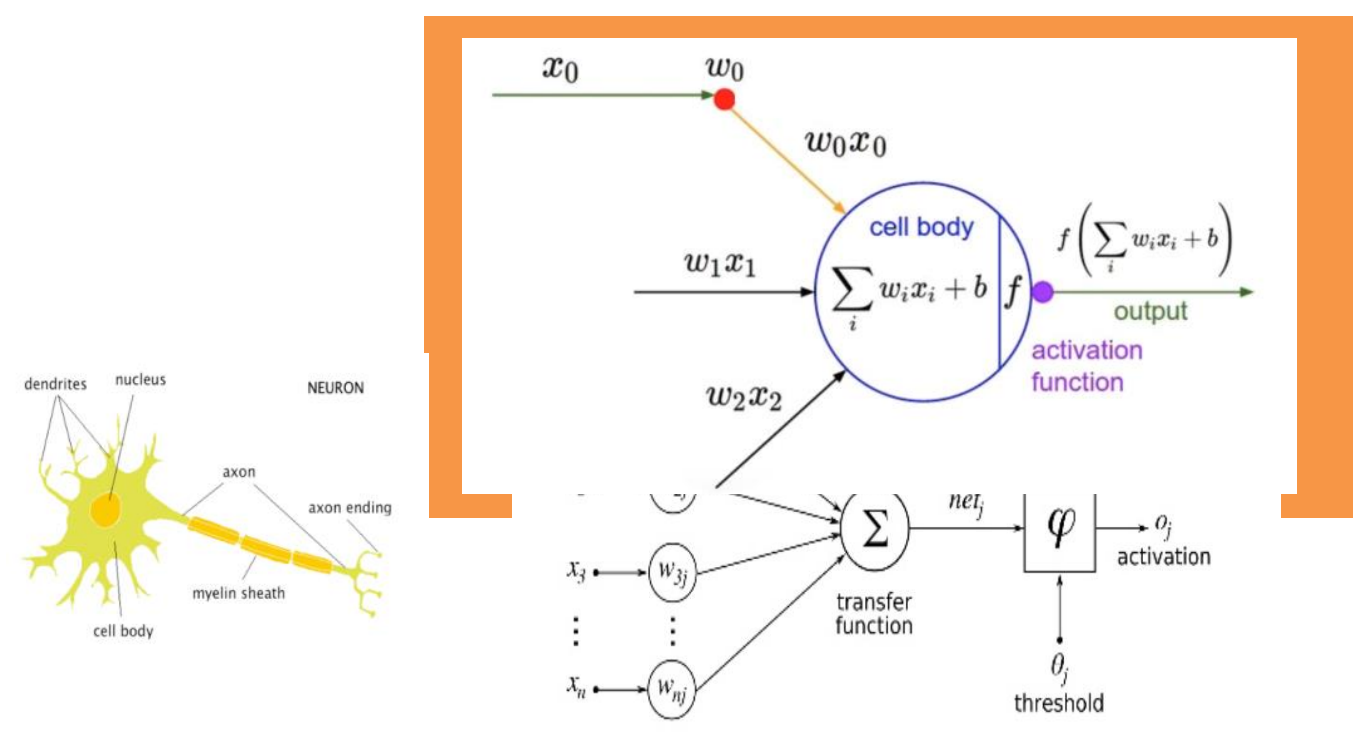

Figure 1. Biological Neuron vs. Artificial Neural Network

\section{Critical Success Factors for the AI Implementation}

There are several CSFs(Critical Success Factors) for the implementation about AI project. First one is top management decision for the AI utilization which is related with business strategy with long term vision. Second one is organization and human resource. Third one is infra structure. Fourth one is support from end users. Fifth one is company strategy. [5]

\subsection{Top management decision}

Top management decision is the most important success factor for the AI implementation. Top management should recognize the value of AI utilization matching business performance. Also, top management must aware the implementation strategy of AI system and AI project roll out plan. [6]

\subsection{Organization and human resource regarding AI project}

Without organization and proper human resource, AI implementation is impossible and end up with failure. Before roll out of AI project, company should set up AI organization and human resource which are AI specialist team who have enough business domain knowledge and AI technology back ground. Not relying with outside consultant, company should develop and keep AI specialist internally with long term vision and perspective view. Also, company should operate change management team of AI project to upgrade and expand the AI project coworking with business end users. [7]

\subsection{Infra Structure about AI system}

For the successful AI implementation, company should invest in infrastructure which include $\mathrm{H} / \mathrm{W}$ and $\mathrm{S} / \mathrm{W}$. Concerning H/W, company should be equipped with Hadoop, Spark, GPU processor and cloud computing. AI related S/W means package and algorithm for the proper AI project. One of the best ways of successful AI implementation is to do the AI project with AI special consulting company which meet project goal with enough consultant members 
who have equipped with business domain knowledge and AI technology as well as project experiences. With the help of open source environment, current algorithm can enhance pretraining, drop-out and rectified unit function. Pre-training function do the pre-train by layer with initialization to avoid over fitting. Drop-out function does the drop out some node in conducting training. Rectified function can replace sigmoid function resulting in decreasing vanishing gradient effect. [5]

\subsection{Suppot from End Users}

Support from end users is one of the most crucial success factors for the AI implementation. In any way end users should be involved in AI project to gather the requirements from them. AI system should be easy to use, reliable and available from end users' perspective points. Also, education about AI system is very important to secure ROI(Return On Investment) with specific end user manuals. [8]

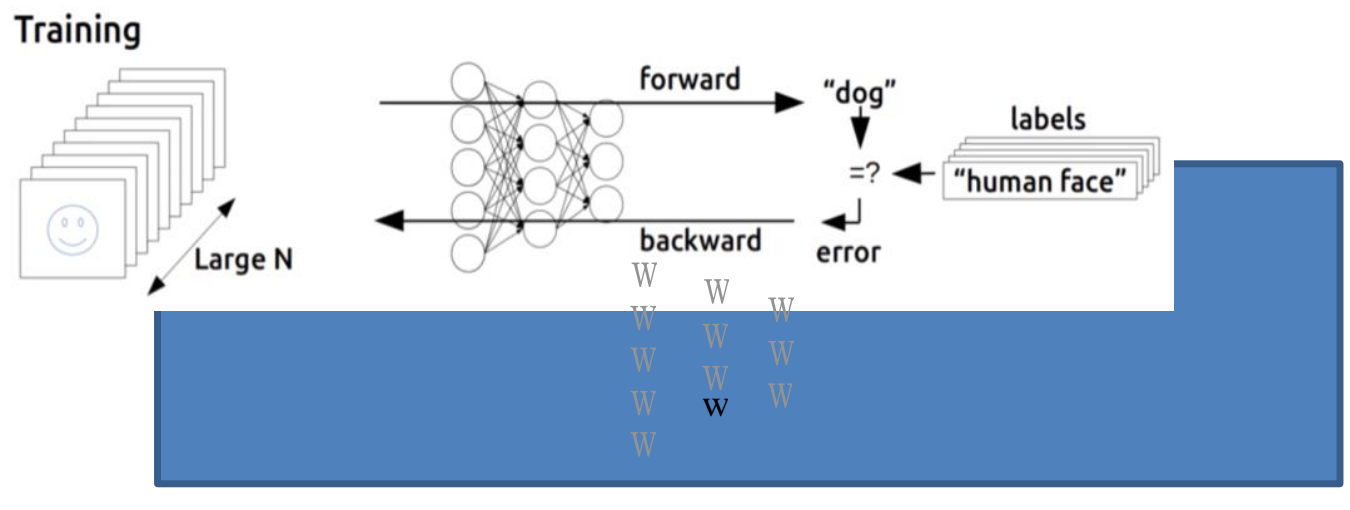

https://devblogs.nvidia.com/parallelforall/inference-next-step-gpu-accelerated-deep-learning/

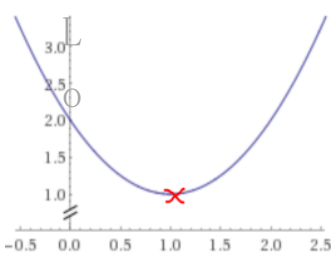

W

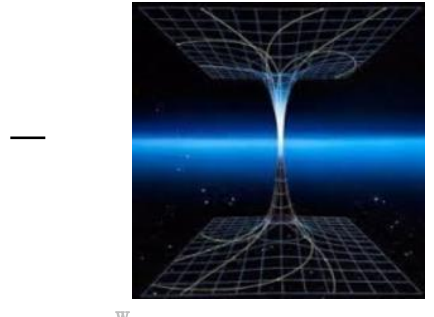

2

\section{Figure 2. Infra about Al Training Flow}

\subsection{Company Strategy}

Along with top management decision, there should be company strategy about AI project roll out plan in connection with investment and company cash flow. Company strategy must include ROI analysis and AI project plan with long term view. Investment in AI system can be one of the competitive edge as company strategy. 


\section{Performance Index for the AI Implementation}

In this study, performance index can be analysis by portion as below table.[9]

Table 1. Performance Index

\begin{tabular}{|c|c|}
\hline \multicolumn{2}{|c|}{ <Major performance index > } \\
\hline \hline Major Index & $\begin{array}{c}\text { Portion } \\
(\%)\end{array}$ \\
\hline Top management decision & 40 \\
\hline Organization and human resource regarding AI project & 20 \\
\hline Infra Structure about AI system & 20 \\
\hline Suppot from End Users & 10 \\
\hline Company Strategy & 10 \\
\hline & \\
\hline Remarks) This index can be different in some company or organization.. \\
\hline
\end{tabular}

\section{Conclusion}

There are several CSFs(Critical Success Factors) for the implementation about AI project. First one is top management decision for the AI utilization which is related with business strategy with long term vision. Second one is organization and human resource. Third one is infra structure. Fourth one is support from end users. Fifth one is company strategy. There are some limitations about this research that should be considered. Firstly, the verification of this conceptual framework about this research model through questionnaire and data analysis needs to be more diversified through other organizations. Secondly, application of this research in different areas such as other corporations could also help for the purpose of extending the validity of these success factors. Thirdly, due to the limitation of time and research work, not all of the various factors are discussed in this study.

\section{Acknowledgments}

Funding for this paper was provided by Namseoul University.

\section{References}

[1] C. D. Scott and R. E. Smalley, "Diagnostic Ultrasound: Principles and Instruments", Journal of Nanosci. Nanotechnology., Vol. 3, No. 2, (2003), pp. 75-80.

[2] Smith, T.F., Waterman, M.S.: Identification of Common Molecular Subsequences. J. Mol. Biol. 147, pp.195197 (1981)

[3] Michael Negnevitsky, Artificial Intelligence, Addison Welsley, (2017)

[4] H. S. Nalwa, "Magnetic Nanostructures", American Scientific Publishers, Los Angeles, (2003).

[5] Big Data Computing Technology, Hanbit Academy pp.14-31 (2016)

[6] IBM project management report, New York (2004)

[7] H. V. Jansen, N. R. Tas and J. W. Berenschot, "Encyclopedia of Nanoscience and Nanotechnology", Edited H.

S. Nalwa, American Scientific Publishers, Los Angeles, vol. 5, (2004), pp. 163-275.

[8] OkGi Kim, Data Science, Ezies Publishing Co, pp. 52-55 (2017) 
Case Study about efficient Al(Artificial Intelligence) Implementation Strategy

[9] J. Kimura and H. Shibasaki, "Recent Advances in Clinical Neurophysiology", Proceedings of the 10th International Congress of EMG and Clinical Neurophysiology, Kyoto, Japan, (1995) October pp.15-19. 